\title{
El latín publicitario ${ }^{1}$
}

\author{
Delio DE MARTINO ${ }^{2}$ \\ Università di Bari “Aldo Moro” y Universitat de València
}

\begin{abstract}
RESUMEN:
El latín, lejos de ser una lengua muerta, revive hoy en día una nueva vida en los textos publicitarios. El gran patrimonio de la literatura latina, sobre todo en Italia, entra en pequeñas o mínimas cantidades en los anuncios impresos, en los spots sea televisivos sea radiofónicos y en las campañas en Internet. No se trata de un mero uso de nuevo del más importante sustrato lingüístico europeo. Como se muestra en el artículo, entrando en diálogo con las lenguas neolatinas, el latín da a la luz expresiones lingüísticas insólitas y nuevas en publicidad, generando un lenguaje que se ha definido con el neologismo "neonlatín", un latín iluminado por el neón de los anuncios.
\end{abstract}

PALABRAS CLAVE: publicidad; latín; neonlatín; motos; César

TITLE: The advertising latin

\begin{abstract}
:
The Latin, far from being a dead language, lives today a new life in advertising. The great heritage of Latin literature, especially in Italy, comes in small or minimum doses in the ads in print, in both radio and television commercials, in campaigns on the Internet. It is not simply a reuse of the most important European linguistic substratum. Entering into dialogue with the Romance languages, Latin creates unusual and new linguistic expressions, generating a language that has been defined with the neologism "neonlatin", a Latin illuminated by the neon of the banners.
\end{abstract}

KEY WORDS: advertising; latin; neonlatin; phrases; Caesar

\section{El latín en la sociedad posmoderna}

A pesar de que sea considerado idioma muerto, el latín sigue sobreviviendo en formas y modalidades diferentes en varios lenguajes en el mundo contemporáneo. Entre éstos además del cine (piénsese en La pasión de Cristo de Mel Gibson), de los cómics, de algunas formas literarias, de Internet (Google y Facebook se pueden utilizar también en latín), quizá el lenguaje más visible sea la publicidad. El latín con su aura de prestigio permite de hecho al texto publicitario emerger en la jungla de mensajes promocionales y regala un toque de esnobismo, incluso cuando se une a la ironía. A pesar de la carencia de estudios en este ámbito, ya en 1925 eran evidentes la utilización del latín en publicidad ${ }^{3}$ y sus peculiaridades.

\footnotetext{
${ }^{1}$ Agradezco la revisión y corrección del texto a la profesora Carmen Morenilla Talens (Universitat de València).

${ }^{2}$ Doctorando en la Università di Bari Aldo Moro (Teoria del linguaggio e scienze dei segni) y en la de Valencia (Lenguas y literaturas), deliode@alice.it.

${ }^{3}$ COTARD, R. (1925): «Le latin dans le réclame», Revue universitaire, XXXIV a. Universitat de València, 216-218.
} 
El latín empieza a difundirse en las diferentes formas publicitarias en el naming, para entrar después en los letreros y finalmente en los spots y en la publicidad en Internet.

Normalmente se trata de vocablos en nominativo, que hacen alarde de enlaces intrínsecos con las cualidades del producto. Podemos ver sobre todo teónimos (la línea de cosméticos Venus), antropónimos del mito y de la historia (Ajax, Amilcar), verbos (Volvo, Fiat), adjetivos (Magnum), sustantivos (la línea de ropa Vestis) y topónimos (Florentia).

Además de estos casos de simple naming, tomados del léxico latino, se observan fenómenos de mutación lingüística conectados a la creatividad publicitaria. Ejemplos son los barbarismos Lancia Phedra y Fiat Ulysse, el solecismo «Nil melior», la aféresis Asus (desde Pegasus) o la palabra compuesta Kia Ophirus, derivada de la unión de Ophir, tierra mítica de bíblica memoria, con el latino rus. Una especie de apofonía contiene la marca Lexus, que deriva de luxus además de aludir a lex ${ }^{4}$.

Se genera así la que Marcello Marchesi ${ }^{5}$, uno de los más celebres autores del Carosello, ha definido, en su divertido volumen Sancta publicitas: 100 flores sententiarum (1970), con el neologismo "lengua neonlatina" o sea derivada del «corrompimento della lingua classica a opera di quella volgare, della cronaca e della pubblicità». El autor con este neologismo se refería a la tendencia lingüística de un tío suyo, cura, de mezclar frases latinas con vocablos de origen publicitaria produciendo expresiones como «Ava Maria», «Fero, fers, tuli latum Fernet»o «In nomine patris Philco et spiritus sancti».

Pero el mismo procedimiento es explotado por la creatividad de los publicitarios, no sólo de los últimos años sino también del principio del siglo pasado. El acrónimo Fiat (Fabbrica Italiana Automobili Torino) es una sutil alusión a sentencias como «Fiat lux» (Génesis 1, 3). Emanuele Cacherano di Bricherasio, uno de los fundadores de la empresa, a quienes preguntaban en torno al nacimiento de la marca respondía «Fiat Fiat et Fiat fuit» mientras los detractores de este naming sostenían que «sapesse troppo d'incenso» ${ }^{6}$. «Desiderare Sorento humanum est» ${ }^{7}$ en la campaña para la Kia Sorento (fig. 1) es una modificación del moto «Errare humanum est, perseverare autem diabolicum ${ }^{8}$.

${ }^{4}$ ChiERICI, S. (29/09/2005): «Latino e automobili di oggi», Virtualcar, http://www.virtual car.it/archivionews/?n=209. Web visitada el 28/1/2012.

5 BAllio, L. y RANOCCHI, A. (2009): Carosello story. La via italiana alla pubblicità televisiva, Roma, Rai Radiotelevisione italiana, 108.

${ }^{6}$ Foriero, E. (1999): Gli automobili. Il lessico delle prime quattro-ruote tra Ottocento e Novecento, Venezia, Marsilio, 42.

${ }^{7}$ De Martino (2011), op. cit., 118.

${ }^{8}$ Cfr. San Agustín, Sermones 164, 10, 14 «Humanum fuit errare, diabolicum est per animositatem in errore manere», Tosi, R. (1991): Dizionario delle sentenze latine e greche, Milano, Rizzoli, 212, sub voce Errare humanum est, perseverare autem diabolicum. 


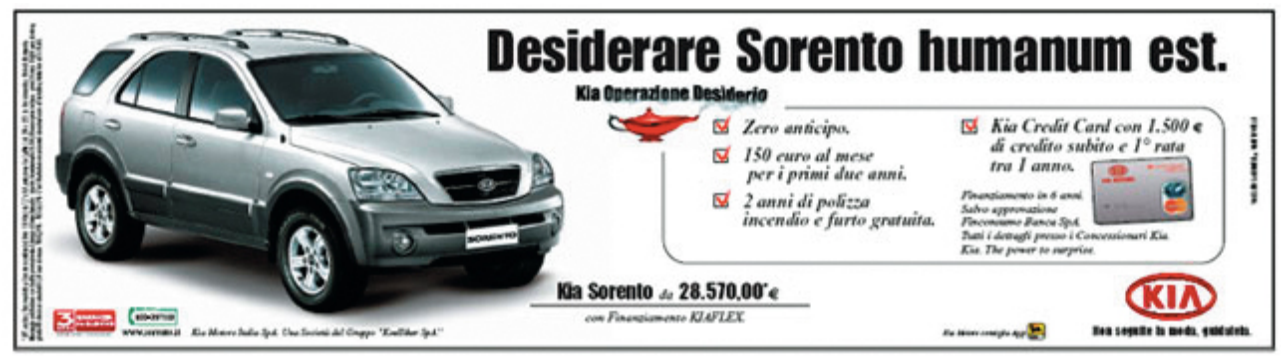

Fig. 1. Anuncio Kia Sorento

«Annuntio vobis novissimi premi» y «Ecce bimbo», eslóganes de la gasolina italiana Api-Ip (2006), son variantes de motos celebres: «Annuntio vobis gaudium magnum», la fórmula de elección del Pontífice y «Ecce homo» (frase de Pilato en el Vangelo de Juan 19, 5). «Quo vadis?» ${ }^{9}$ de la misma campaña es una citación directa de la pregunta de Pedro a Jesús en los Actos del martirio de Pedro de Pseudo-Lino (6).

Además de ejemplos de latín religioso otros casos son el nombre de la fonda Rex's, donde el nominativo se une al genitivo sajón inglés, o el latín inventado de Banania $^{\mathbf{1 0}}$, y el nombre mixto (inglés-latín) de la compañía de aviación española Air nostrum ${ }^{11}$. Pero al neonlatín son traducidos, además de lemas particulares, también motos latinos.

Sea como fuere, se decida o no usar nombre o un eslogan mítico, las lenguas clásicas son un bagaje sedimentado en la cultura que las empresas deben tener en cuenta para evitar peligrosos "falsos amigos". Muy poca atención ha demostrado Kia no adaptando al mercado italiano el nombre del monovolumen Carens. El nombre deriva de un acrónimo compuesto por Car y Renaissance y tenía el objetivo de mostrar la nueva línea de la Kia, pero en Italia no puede evitar recordar el participio presente de careo («manco») y constituye entonces un clásico ejemplo de "falso amigo" que hay que evitar.

${ }^{9}$ Tosi (1991), op. cit., 679, sub voce Quo vadis? Un «Quo vadis sciatore?» más antiguo se encuentra en un anuncio italiano de 1947 de la región Trentino, cfr. GRASSI (2010), web cit.

${ }^{10}$ CotaRd (1925), op. cit., 217.

${ }^{11}$ De Martino, F. (2010): Antichità \& pubblicità, Bari, Levante, 694. La sección "Parole antiche" (693-725) contiene varios otros ejemplos de naming latín: Aequilibrium, Alma plena, Anima mundi, Aurum, Clivus, Plus, De gustibus, Divinus, Domus, Felix, Fidelis, Focus, Fulvia, Gaudium et spes, Immensum, Insignia, Lumina, Lacryma Christi, Lupus in fabula, Millennium, Et nunc, Oppidum, Omnia, Puer Apuliae, Quantum, Quo vadis, Sine pari, Stupor mundi, Terra maiorum, Turris, Trebius Valens, Valor, Vivarium novum. 


\section{Los motos latinos y el naming}

Los motos latinos, incorporados a la cultura y al imaginario colectivo, son una preciosa fuente de inspiración para los publicitarios. Además la sententia y el eslogan comparten características estructurales semejantes, como son la brevedad, la concisión y sobre todo la capacidad de impactar al lector e imprimirse en la memoria, tanto que la sententia se ha considerado un prototipo de la moderna publicidad ${ }^{\mathbf{1 2}}$.

Ya dentro del estricto ámbito del naming una parte del latín publicitario está constituido por logos que aluden a célebres motos latinos.

Audi, por ejemplo, es una marca automovilística que deriva de la traducción al latín del patronímico, o sea del apellido del fundador August Horch que por problemas legales se vio obligado a cambiar el nombre de su empresa. De hecho Horch, en alemán es el imperativo «escucha», y corresponde al latín Audi. Pero tras esta operación se esconde un velado moto latino. La August Horch Automobilwerke gmbH cambió de nombre gracias a la sugerencia de un pariente del fundador, estudiante de latín, que tuvo la idea inspirándose en el moto «Audiatur et altera pars». La frase, que forma parte del lenguaje jurídico y está a la base del "principio de contradicción", por el que en un juicio es preciso escuchar también a la parte contraria, fue acuñada en época medieval pero refleja sentencias de época latina. De todas maneras en el moto se puede leer el eco de textos literarios clásicos entre los que la Medea de Seneca ${ }^{13}$ : vv. 199-200 «Qui statuit aliquid parte inaudita altera / aequum licet statuerit, haud aequus fuit» ${ }^{14}$.

El naming del modelo Astra de Opel trae a la memoria el moto latino «Per aspera sic itur ad astra», cuyos arquetipos están en varios autores a partir de Hesíodo (Trabajos y días 289 s.) y sobre todo Seneca (Hercules furens v. 437 «non est ad astra mollis e terris via» en el acto II) y Virgilio («sic itur ad astra» Eneida 9, 641). Del mismo modo el Renault Modus recuerda el celebre «Est modus in rebus» de las Sátiras de Horacio (I, 1, 106).

Otras veces un acrónimo de una frase latina puede generar un logo, como en el caso del profiláctico italiano Hatù, derivado del moto latino «Habemus tutorem», a su vez irónica adaptación de la formula con la cual el Cardenal protodiácono proclama la elección del papa $^{15}$ : «Habemus papam». Otro ejemplo es la marca Asics,

12 SPINA, L. (1993): «La sententia nell'epoca della sua riproducibilità tecnica», en Pennacini, A. (ed.), Retorica e comunicazione: teoria e pratica della persuasione nella società contemporanea, Alessandria, Dell'orso, 153-166.

13 Tosi (1991), op. cit., 514, sub voce Audiatur et altera pars.

14 De Martino, D. (2012): «El logos femenino en la publicidad mitológica», en De Martino, F. y Morenilla Talens, M. (eds.), Teatro y sociedad: El logos femenino en el teatro, Bari, Levante, 325-348.

15 Tosi (1991), op. cit., 680-681, sub voce Habemus papam. Un «habemus sugo» está en una publicidad de la salsa De Cecco, cfr. Grassi, G. (2010): «Un'acqua ad hoc», Alipes. Arte e cultura nella pubblicità, http://www.italipes.com/artelatino.htm. Web visitada el 28/1/2012. 
derivada del acrónimo «Anima sana in corpore sano», variación del conocido moto «Mens sana in corpore sano» tomado de Juvenal (Sátira 10, 356) ${ }^{\mathbf{1 6}}$.

Particularmente predispuestas a un uso de las lenguas clásicas están las marcas tipográficas que presentan a menudo un moto latino: la Paravia (famosa editorial escolar italiana), por ejemplo, se precia de un árbol con un cartucho con la sentencia «In labore fructus». Este logo contiene una adaptación simplificada de los famosos árboles medievales de la ciencia que representaban la organización y la unificación del saber universal.

\section{Los motos de César}

César es el comandante romano por antonomasia y a él se vincula gran parte de las frases latinas que aparecen en los carteles y que son pronunciadas en los spots.

La forma de citación de frases celebres más común es la que Genette llama intertextualidad ${ }^{17}$ o bien «la presencia efectiva de un texto en otro». La más frecuente entre todas es el celebérrimo moto "Veni Vidi Vici» frase ya nacida con un fin propagandístico, ya que según Plutarco (Vida de César 50, 3-4) fue transmitida por César a un mensajero llamado Macio para comunicar a Roma la victoria de Zela sobre Farnaces $\mathrm{II}^{18}$. El uso publicitario del moto es aún más evidente en el testimonio de Suetonio, el cual en la Vida de César cuenta $(37,2)$ que fue escrito en una enseña durante el triunfo póntico propio como un moderno cartel publicitario: «Inter pompae fercula trium verborum praetulit titulum "Veni, vidi, vici" non acta belli significantem sicut ceteris, sed celeriter confecti notam». Como indica Suetonio, la frase tuvo un éxito enorme en época antigua porque comunicaba eficazmente la idea de rapidez, gracias a la estructura de tres miembros asindéticos, unidos por la aliteración, por el homeotéleuton y por el isosilabismo.

No asombra, pues, que el moto ya a principios del siglo pasado fuera utilizado en publicidades de automóviles ${ }^{19}$. Por ejemplo el anuncio de Romano del 1928 para la Fiat contiene el headline: «La nuova 6 cilindri Fiat mod. 520 sta destando unanime ammirazione in tutto il mondo. Veni Vidi Vici». Naturalmente las condiciones histórico-políticas de la dictadura fascista promocionaban un uso del latín con un significado muy diferente respecto a hoy.

Variaciones en el tema se hallan sobre todo con la substitución de «Vici» que crea un irónico aprosdoketon y que en parte sigue un modelo utilizado por Tasso

\footnotetext{
${ }^{16}$ Agradezco la indicación a la profesora Carmen González (Universidad Autónoma de Madrid). Otro naming que alude a este moto es SanaGens, empresa italiana de calzados fisiológicos. El eslogan «Mens sana» se encuentra en un anuncio de Liabel, cfr. GRASSI (2010), web cit.

${ }^{17}$ Genette, G. (1982): Palinsesti. La letteratura al secondo grado, Torino, Einaudi, 1997, 4.

${ }^{18}$ Tosi (1991), op. cit., 434, sub voce Veni Vidi Vici.

19 De Martino, D. (2011): «Io sono Giulietta». Letterature \& miti nella pubblicità di auto, Bari, Levante, 116, 118. Cfr. GRASSI (2010), web cit.
} 
en la Gerusalemme liberata, donde con una anadiplosis es replicado el «vidi» 18, 40, 5-7: «A quel temuto / bosco n'andai, come imponesti e '1 vidi / vidi e vinsi gli incanti ${ }^{20}$. Sirva de ejemplo un moderno cartel del Bmw Z3 (1998) en el que se lee «Veni. Vidi. Veni» con la repetición de un miembro (fig. 2). Otro caso es el eslogan «Veni vidi parking» (2012), del cine Galleria de Bari (fig. 3). Por el contrario, la substitución de «Vici» por una imagen está en el origen de un cartel de 2003, en el que un Mercedes clase $\mathrm{S}$ toma el lugar del último miembro del moto (fig. 4).

La dilución del moto, como en Tasso, es un elemento verificable también en la campaña moderna de la Tim (Telecom Italia Mobile, la mayor compañía telefónica italiana de móviles), insertada en la serie La storia d'Italia secondo Tim, realizada en 2011-2012 para conmemorar los 150 años de la unificación de Italia a través de una relectura publicitaria de la historia del país. En un spot de hecho el general César anuncia:

«Veni, vidi, vici. Veni, vidi er tempo de oggi, vidi la posta elettronica, chattai con Cleopatra, pubblicai le foto di guerra su Facebook, e vici».

En Italia el moto generalmente no es traducido a causa de su notoriedad y sacralidad, pero en otros países se traduce sin demasiados escrúpulos. Ya a principios del siglo el reclame de Bibendum de la Michelin (mascota cuyo nombre deriva de una citación, vd. infra) en versión gladiador citaba la traducción francesa del moto de César. En Italia, a pesar de que no se haya traducido literariamente, el moto aparece en traduciones neonlatinas-macarrónicas. «Vieni guidi vinci», por ejemplo, era el nombre de una campaña del 2006 para la recogida de puntos de la marca de gasolina Api-Ip subtitulada La promozione che farà storia (fig. 5). Otra paronomasia es el eslogan «Siedi, Vedi, Vivi» de 2010 de la cadena de productos electrónicos Trony (fig. 6). De todas maneras el moto es utilizado también por las empresas extranjeras como demuestran el logo de Philip Morris (fig. 7), la empresa productora de los cigarrillos Marlboro, o en ámbito militar el logo de la US Army Sniper School.

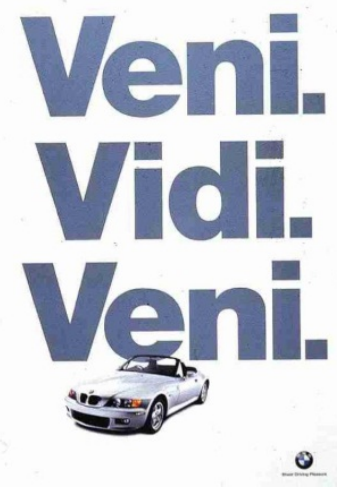

Fig. 2. Anuncio Bmw Z3

\section{veni (O) vidi P parking}

Fig. 3. Eslogan del cine Galleria Bari

${ }^{20}$ Tosi (1991), op. cit., 434, sub voce Veni Vidi Vici. 


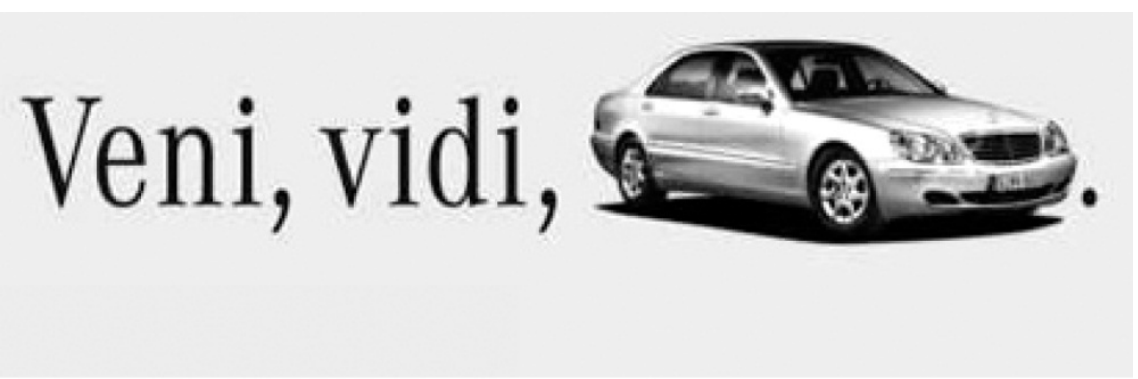

Fig. 4. Anuncio Mercedes clase $S$

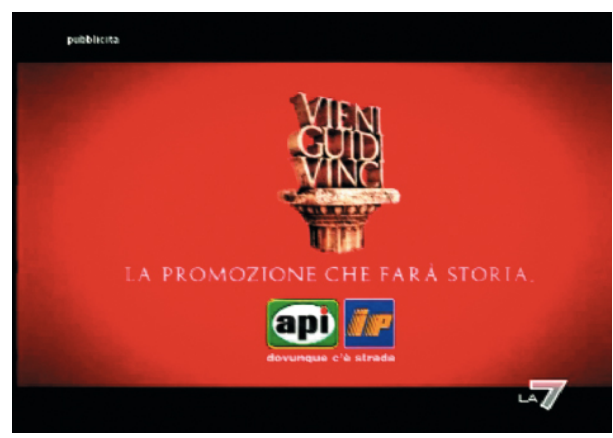

Fig. 5. La promozione che farà storia

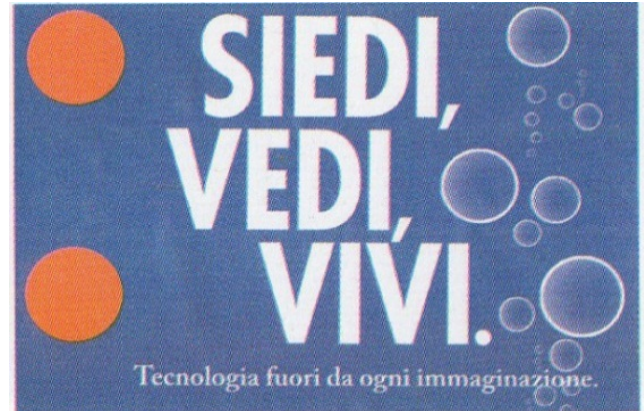

Fig. 6. Papel volante Trony

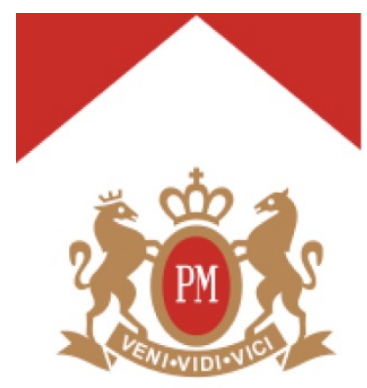

Fig. 7. Logo Marlboro

Además de «Veni Vidi Vici», otra sentencia de César que ha entrado en el ámbito publicitario es «Alea iacta est», frase pronunciada según Suetonio (Vida de César 32) por César en 48 a. C., en el momento de cruzar en armas el Rubicón (límite que no se debía traspasar con equipo militar) para combatir contra Pompeo e iniciar la guerra civil. A diferencia del «Veni vidi vici», «Alea iacta est» frecuentemente es traducido con modificaciones lingüísticas, como en una publicidad de 
Telecom Italia «Il cubo è tratto» o en el reciente spot de la Tim (2011) donde el dado se convierte en un dado de sopa.

En fin, cabe recordar también el famoso «Tu quoque, Brute, fili mi?», transmitido por Suetonio (Vida de César 82) y hecho célebre por Shakespeare en su tragedia. En un spot de los cereales Weetabix de 1994 la frase «Et tu brute?» es pronunciada en un contexto subvertido, ya que Bruto ${ }^{21}$ es arrestado antes de que asesine a César. El anulamiento paródico de la calidad de trágico es evidente también en otro video de la serie La storia d'Italia secondo Tim (2011), en el que César pronuncia «Tu quoque» cuando es golpeado por Bruto, aunque esto sucede durante una batalla con pistolas de agua. Traducido al inglés, en cambio aparece en el eslogan de la campaña Fiat titulada Julius Caesar ${ }^{22}$ contra el uso del móvil durante la conducción: «If you want your last words to be the same as Julius Caesar's simply text "Even you Brutus?" while you drive».

El nombre de César, aunque referido al título de César y no al propio César, aparece también en la frase "Ave Caesar, morituri te salutant», presente como eslogan en algunas publicidades (entre ellas una de $\mathrm{Nike}^{23}$ ) y transformada en neonlatín en expresiones corrompidas por el dialecto romanesco como «Ave no' Nerone te saluta» (frase pronunciada por un Nerón niño en el spot italiano de los congelados Quattro salti in padella Findus rodado en 2003 por el popular director de cine Gabriele Muccino) o «Morituri te saluta», pronunciada por César en el spot Tim del 2011.

\section{Los motos de Horacio}

Inmediatamente después de las citas que se refieren a a César, las más utilizadas son las frases procedentes de los escritos de Horacio. El caso más conocido de campaña publicitaria ligada a una oda de Horacio es aquel de Bibendum, el histórico emblema de Michelin.

\subsection{Bibendum y su metamorfosis}

Multiformes enlaces con la literatura presenta uno de los mitos publicitarios más longevos y famosos en la industria del automóvil: Bibendum, conocido también como "hombre Michelin". El primero y más evidente signo literario es la onomástica latina, que se remonta al origen de su más que centenaria historia. En 1894 los hermanos André y Édouard Michelin, inventores del revolucionario neumático desmontable, visitando su stand en la Exposición Universal y Colonial de Lión,

\footnotetext{
${ }^{21}$ Un anuncio con Bruto y César enteramente en latín es el del zumo de fruta Rybb \& Decker de 1999.

${ }^{22}$ www.coloribus.com. Web visitada el 28/1/2012.

${ }^{23}$ De Martino (2010), op. cit., 712.
} 
observaron unas pilas de neumáticos de diversos diámetros con una forma vagamente humana. Pensaron entonces que añadiendo brazos y piernas se podría crear un muñeco para utilizar como testimonial/representación del producto. Poco tiempo después encontraron al artista O'Galop, pseudónimo de Marius Rossillon, que les enseñó algunos bocetos publicitarios entre ellos uno realizado para una cervecería de Mónaco. El dibujo ilustraba una especie de Gambrinus ${ }^{24}$, legendario rey de las Fiandras, patrón de la cerveza, que levantaba una copa de cerveza acompañado por el headline «Nunc est bibendum», citación del primer verso de una oda de Horacio con la que celebra la muerte de Cleopatra $(I, 37,1)$ y a su vez inspirada en el fragmento de Alceo [vṽv $\chi \rho \tilde{\eta} \mu \varepsilon \theta v ́ \sigma \theta \eta v]$ (fr. 332 Voigt). André Michelin tuvo entonces una gran intuición: juntar el dibujo de O' Galop, la idea de un hombre hecho enteramente de neumáticos y el eslogan horaciano.

A partir de esta intuición O'Galop realizó en 1898 el afortunado cartel que marcó el nacimiento del "hombre Michelin". En el visual el emblema de la marca levanta una copa llena de fragmentos de botellas y clavos para brindar acompañado por el headline «Nunc est bibendum!!.. C'est-à-dire à Votre Santé le pneu Michelin boit l'obstacle» («Nunc est bibendum!!..., o sea a la vuestra salud el neumático Michelin se bebe el obstáculo») (fig. 8). En esa época este hombre todavía no tenía un nombre oficial, pero en julio del mismo año, durante una carrera Paris-AmsterdamParis, el público viendo llegar a André Michelin, empezó a gritar «Llega Bibendum». A partir de entonces el simpático muñeco fue bautizado con el gerundivo latín Bibendum, retomado del primer cartel. Desde su primer aparición Bibendum ha ido cambiando de aspecto y lleva ya más de un siglo apareciendo en innumerables versiones, algunas de ellas vinculadas a la literatura. En otro cartel de 1905 es dibujado como un gladiador en la arena del Coliseo que ha vencido a otro hombre. Con una rueda usada como escudo domina el cuerpo del vencido, sobre cuya sombra está impresa la letra «Pollice verso». Otro texto, como un comentario, explica la situación: «La foule acclame Bibendum et condamne ses concurrents». Pero se añade también otra citación literaria de César en el headline: «Ave Bibendum. Tu es venu, tu as vu, tu as vaincu, grâce a l'exerciseur Michelin». En otras variaciones Bibendum aparece, en cambio, disfrazado de Hércules o Arquímedes. El muñequito, pues, se convirtió rápidamente en un verdadero personaje, cargado de fuerza literaria y capaz de volverse protagonista de diversas historias. Los mismos hermanos Michelin pronto se dieron cuenta de esta metamorfosis de su criatura como subraya una declaración de André: «El bueno de Bibendum ha sido desde entonces reproducido en muchas posturas y circunstancias diversas. Se le han añadido muchas frases. Es decir, tiene una personalidad bien clara, muy acusada. Como una

${ }^{24}$ DARMON, O. (1997): El gran siglo de Bibendum, Paris, Ediciones Hoëbeke, 22-23. 
creación literaria, habita el espíritu de las masas... Y reconozco que su éxito, que ha contribuido en gran medida al de nuestra casa, me llena de orgullo paternal» ${ }^{25}$.

Por otra parte, Bibendum a principios del ' 900 entró también en la historia literaria. En España Ramón Gómez, traductor español del manifiesto futurista, escribió un cuento titulado ¿Qué quiere usted ser?, incluido en el libro Gollerías (editorial Sempere, Valencia, 1925). En el cuento un periodista preguntaba al autor qué le hubiera gustado ser ser y éste contestaba «Yo, neumático Michelin» y después de una petición de aclaración del periodista, decía: «¿Es que no ha visto usted esos anuncios que consagran al Michelín como un neumático feliz, orondo, sin puñalada trapera, nalgado por todos los sitios y fumándose un gran puro siempre? Todo asegura que el Michelin es un superviviente eterno y que camina sin cansarse por todo el mundo; todas sus heridas se cicatrizan y todos sus gestos están llenos del orgullo duradero» ${ }^{26}$.

Además de la narrativa y del arte Bibendum parodiaba también la literatura teatral: en 1911 Michelin empezó a publicar anuncios en la cubierta trasera de L'illustration théâtrale, suplemento de L'illustration. Aquí Bibendum subía a un palco escénico para promover los neumáticos vestido como actor teatral ${ }^{27}$.

En fin Bibendum fue también el nombre de una importante revista corporativa de estilo literario de Michelin (fig. 9): una de las cubiertas más originales, que testimonia la relación con las diversas artes, es la del 1926 en que el hombre se convierte en la parodia de La primavera de Botticelli.

Pero, más allá de Bibendum, otros fragmentos de composiciones horacianas han sido elegidos por los copywriters. El mismo «Nunc est bibendum» era utilizado en otras publicidades como en el sitio Internet de la empresa italiana de gasolina Api-Ip en el ámbito de la ya citada promoción Vieni guidi vinci. El moto «Carpe diem» tomado de las Odas (I, 11,8) y en Italia hecho celebre por el film de Weir L'attimo fuggente, revive en muchos anuncios ${ }^{28}$ y se encuentra en la reciente campaña italiana de la Mercedes clase $\mathrm{C}$ traducido y modificado en la versión automovilística «Guida l'attimo» (2010).

Pero aún más irreverente es la adición de la "s" en el nombre de la empresa de calzados Scarpe Diem. Finalmente el «Est modus in rebus» tomado de las Sátiras (I, 1, 106) se lee en un cartel del 2004 del Renault Modus cuyo naming ya sugiere un enlace con la sentencia del escritor de Venosa (fig. 10).

${ }^{25}$ Devynck, T. (2005): «Bibendum y los suyos”, en AA.VV., «Nunc est bibendum!!..», Un mito gráfico desde 1898, Michelin España Portugal, 24.

${ }^{26}$ PÉREZ, C. (2005): «Bibendum y las vanguardias (algunas consideraciones)», en AA.Vv. (2005), op. cit., 10-11.

${ }^{27}$ DARMON (1997), op. cit., 47-48.

${ }^{28}$ De Martino (2010), op. cit., 698-699. Sobre la pervivencia literaria del Carpe diem cfr. Fo, A. (2008): «Modi oraziani di pensare il tempo: tratti della fortuna moderna del carpe diem e di altri spunti delle Odi», en AudAno, S. (ed.), Aspetti della Fortuna dell'Antico nella Cultura Europea. Atti della quinta giornata di studi, Sestri Levante, 7 marzo 2008 per Emanuele Narducci. Pisa, Edizioni ETS, 61-107. 


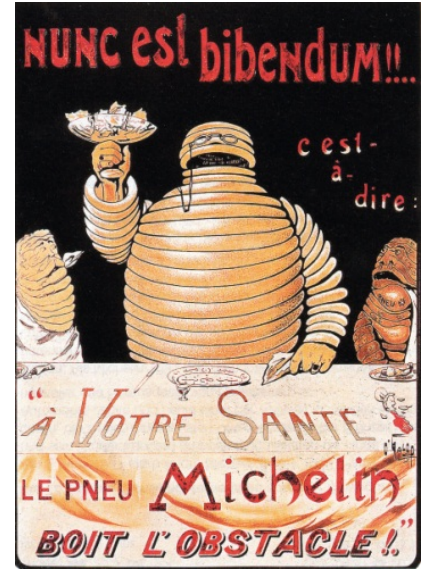

Fig. 8. Anuncio Bibendum

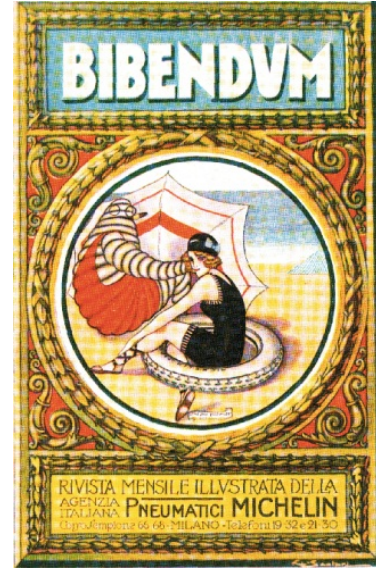

Fig. 9. Revista Bibendum

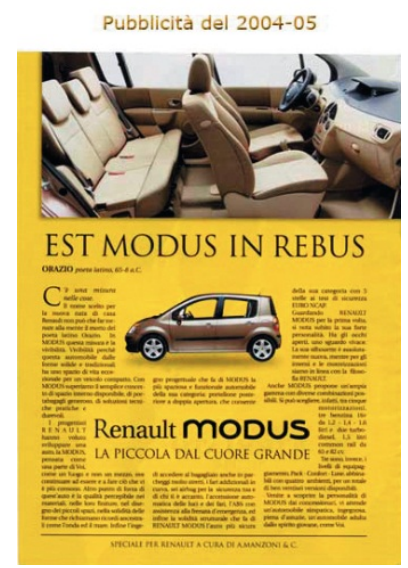

Fig. 10. Anuncio Renault Modus

\section{Otros autores y modos de decir}

El panorama del latín publicitario más allá de las categorías citadas realmente es mucho más variado y comprende autores como Lucano ${ }^{29}$ o Catulo, que ha entrado además en el naming de diversos bares y cafeterías, y también en una valla de hace unos años cerca de Salerno: «Cenabis bene, mi Fabulle, apud me paucis / si tibi favent, diebus (...) Catullo Carmina XIII. Perché a tavola si ferma il tempo» ${ }^{30}$. Pero a menudo las frases proverbiales no se citan completas. El moto medieval «Verba volant» aparece en un folleto de un e-book reader de Sony (fig. 11). La continuación «Scripta manent» está en cambio en el anuncio de 2011 del teléfono móvil Galaxy de Samsung (fig. 12). Además de estas citas identificables, podemos encontrar palabras sueltas o pequeños grupos de palabras muy conocidas y que no se pueden atribuir a un autor en particular, como «Aeternum», «Magnum», «Optimus», «Optimum», «Festina», «Gaudium et spes», «Hic sunt leones», «Imaginarium», «Rex», «Simposium», «Verbatim», «Vicinum est», «Claudia Domina aquae» $^{31}$. Incluso para productos populares se ha hecho esta elección, como para los bocadillos Mc Donald: en la campaña promocional de 2000 se han utilizado eslóganes latinos como «Nec plus ultra», «Irresistibilis divinus», «Mc Roma bonus est» $\mathrm{y}$ «Incomparabilis delicius». En el sector de la comida rápida se descubre también el bocadillo «El Summum» de la cadena Pans \& Company ${ }^{32}$. Otros ejemplos que podemos aducir, son la línea de vestidos «Ad Hoc», la boutique barese

\footnotetext{
${ }^{29}$ GRASSI (2010), web cit.

${ }^{30}$ De Martino (2010), op. cit., 636.

31 Grassi (2010), web cit.

${ }^{32} \mathrm{http}$ //www.pansandcompany.com/ing/navidad.html. Web visitada el 28/1/2012.
} 
«Amanda puella», aún en Bari, el pub con el nombre del moto latino «In vino veritas», la lavandería «Candidus», la trattoria «Intra moenia» en el centro histórico de Oria (Brindisi).

Un eslogan en latín no referible a ningún autor es también aquel del anuncio del Omino Bianco realizado como un mosaico pompeyano: «Quieti Estote. Tuto maculas tollet» (fig. 13).

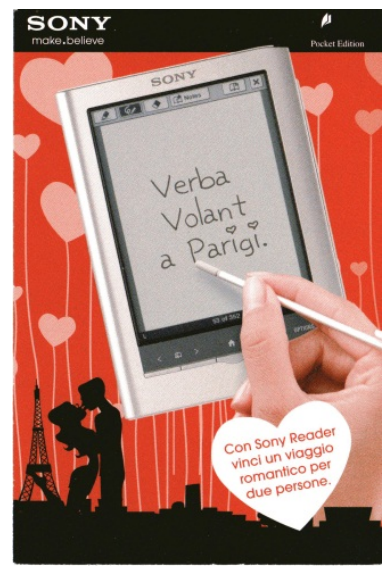

Fig. 11. Folleto Sony

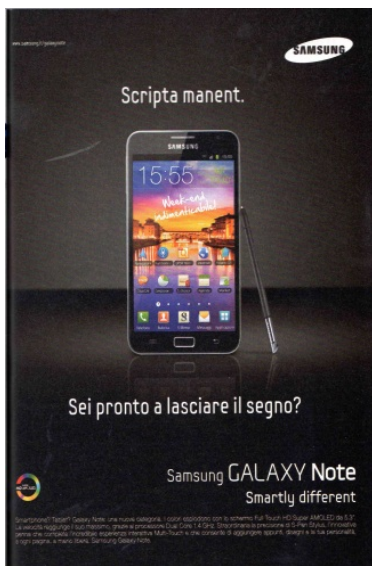

Fig. 12. Publicidad

Samsung

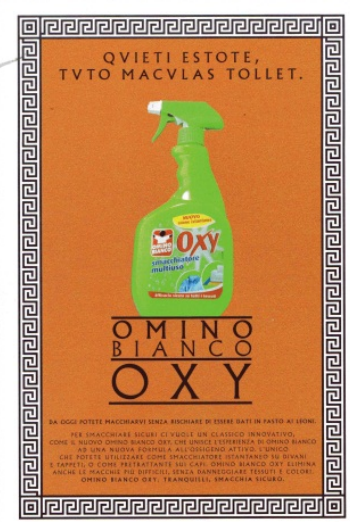

Fig. 13. Publicidad

Omino Bianco

El latín es una de las asignaturas fundamentales en los licei classici y en los licei scientifici italianos y el recuerdo de su enseñanza ha entrado en la publicidad. En 2009 un divertido spot de la empresa telefónica Tim (Telecom Italia Mobile) ponía en escena un coloquio con la profesora de latín, interpretado por Christian De Sica en el papel del padre de un alumno y por Belén Rodríguez en el rol de la docente de latín. Estos dos actores son muy conocidos en Italia por haber actuado en películas de un género típicamente italiano llamado Cinepanettone dirigido a un público medio nacional: se trata de comedias ligeras estrenadas en Navidad y basadas en juegos de palabras, traiciones amorosas y situaciones grotescas y con una suave connotación erótica. La campaña intenta recrear la atmosfera de estos filmes creando juegos de palabras en latín. De hecho en el video publicitario De Sica está buscando a la profesora de latín del hijo y hablando por el móvil se queja a su mujer de ese encargo, hasta que descubre que la profesora es la bella y fascinante Belén Rodríguez. Para ligar con la joven profesora, finge estar interesado en el latín citando el De bello gallico y empieza a inventar palabras en latín. Se presenta como el pater de Carlo y acuña el moto «Pater istruitus filius purem». Le pide entonces el número de teléfono para "latinizzare", pero la profesora explica que el hijo tiene problemas con la perifrástica, palabra de la que el padre ignora el significado. El guión está en perfecto estilo neonlatín: 
De Sica al teléfono: «Col professore di latino ci parlano le madri, ma poi chi lo parla più il latino?».

De Sica se da cuenta de Rodríguez: «Ma certo che ho comprato il De bello gallico. Ti devo salutà. Piacere io sono il pater di Carlo».

Rodríguez: «Ah molto lieta. Suo figlio è molto bravo in latino».

De Sica: «Pater istruitus filius purem. Piuttosto Lei mi dia il suo numero di telefono che latinizziamo. Noi due senza limiti».

Voz en off: «Tim per 2. Telefonate sms senza limiti con il tuo numero Tim preferito».

Rodríguez: «Però suo figlio con la perifrastica...».

De Sica: «Eh la perifrastica...».

Existe una segunda versión reducida a 15“ del spot donde siguen los juegos de palabras. La profesora dice a De Sica que él aparece como un Deus ex machina y éste, malinterpretando, le responde que acaba de hacer la revisión de su vehículo y pide el número di teléfono «per latinizzare»:

Rodríguez: «Perché lei si pone come un Deus ex machina...».

De Sica: «Deus ex machina? Ho appena fatto il tagliando. Piuttosto Lei mi dia il suo numero di telefono che latinizziamo».

La misma empresa ha regresado a este tema de la enseñanza en 2011 con una serie publicada en Internet y denominada Corso di romanità. Se trata de un curso impartido por Marcantonio para preparar a Cleopatra para su llegada a Roma y que es dirigido también al espectador. El curso multimedial prevé la respuesta a unas simples e irónicas preguntas para verificar el aprendizaje. El ciclo se inicia con un curso de lengua romana, que anacrónicamente no es un verdadero curso de latín, sino de dialecto romanesco. Dentro de la misma campaña, titulada La storia d'Italia secondo Tim, se escucha un monólogo con un amplio muestrario del latín que ya ha entrado en el vocabulario común y mezclado con el romanesco. El popular actor Neri Marcorè con túnica de César habla por teléfono con una bella romana (fig. 14):

«Ma scusa bella puella come sarebbe a dire che non mi conosci bene? Io c'ho un $\mathrm{cu}$ rriculum di tutto rispetto, c'ho un modus operandi che levete. E su, e dai, che dobbiamo aspettare? Il post-mortem? Non lo sai che il tempus fugit? Carpe diem, no? Ah ce stai. Oh deo gratias. Allora come famo? A che ora ce vedemo? Hic et nunc? A priori? A posteriori? Ad maiora? Ad personam? Perfetto. Dai vedrai che ce divertimo ad libitum. Eh in vino veritas. Saecula saeculorum. Si capisce. Ave e quando dico ave ho detto ave... La prossima. Ave te ricordi di me?». 


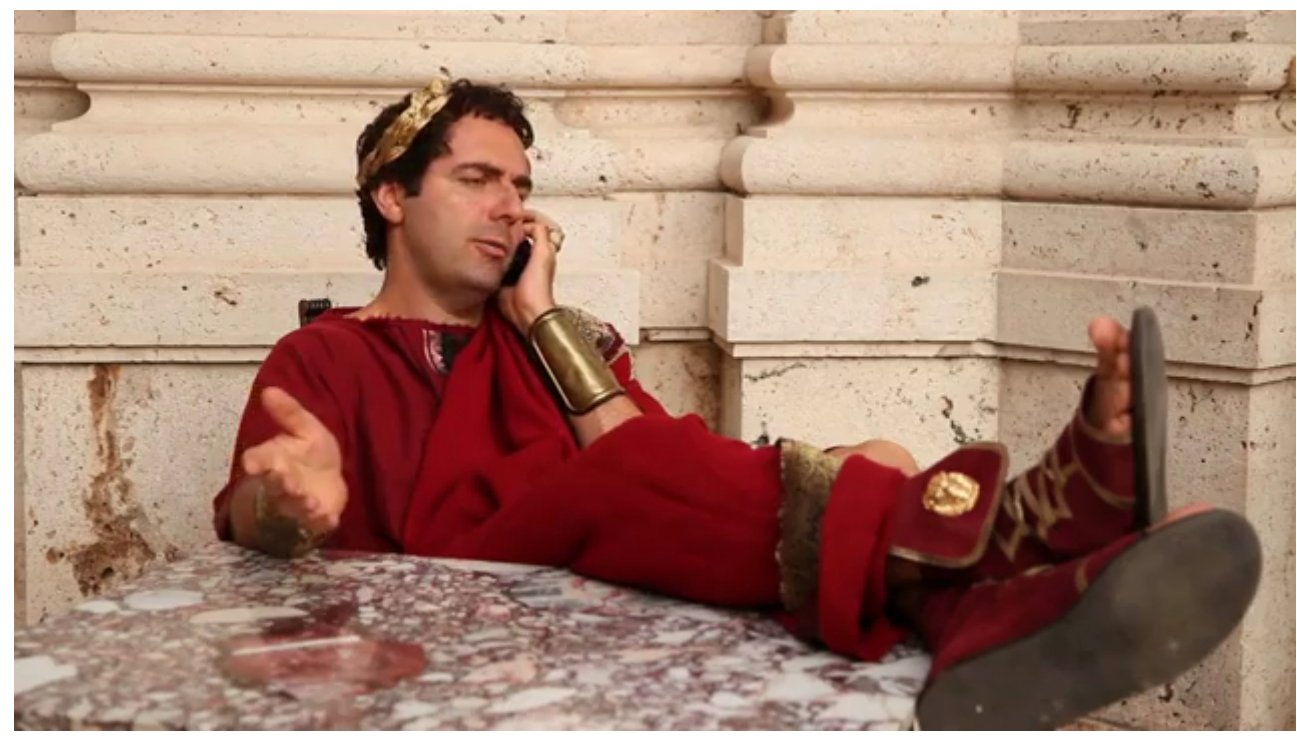

Fig. 14. Spot La storia d'Italia secondo Tim: César

\section{Conclusiones}

Muchas historias de la publicidad hacen remontar las primeras formas de publicidad a las enseñas latinas halladas en Pompeya $^{33}$, e individúan las primeras formas de eslogan políticos en las inscripciones murales pompeyanas. Pero omiten el hecho de que en las más modernas estrategias publicitarias el latín vuelve a emplearse. El enorme patrimonio de la literatura y de la historia romana es de hecho subdividido, partido y desmembrado por la publicidad y de éste se reutilizan las más celebres expresiones en un anacrónico potpourri lingüístico. En este proceso el lenguaje publicitario involuntariamente se trasforma en vehículo cultural, en una especie de publicidad social, que renueva la memoria de una historia y de una literatura aprendida en escuela y de este modo se promueve su profundización. Además de la simple inserción de lemas o expresiones descontextualizadas en ambientes o situaciones nuevas, en la publicidad el latín se mezcla con el lenguaje más moderno.

Esta utilización del latín en el campo comercial publicitario renueva la lengua muerta reavivándola y poniéndola en una condición de diálogo metahistórico con el italiano, el inglés y a veces con otros idiomas. La contaminación lleva a crear enlaces inusitados y creativos entre el italiano y el sustrato latino renovando su

${ }^{33}$ Abruzzese, A. y Colombo, F. (ed., 1994): Il dizionario della pubblicità. Storie tecniche personaggi, Bologna, Zanichelli, 18 sub voce Antichità, pubblicità nella. Mas las primeras formas publicitarias se hallan ya en la antigua Grecia, cfr. DE MARTINo (2010), op. cit. 
significado. Se genera, pues, la bajtiniana hibridación o bien «la mezcla de dos lenguajes sociales dentro de los límites de un solo enunciado, un encuentro, al interior de la arena del enunciado entre dos conciencias lingüísticas separadas entre sí por una época, por diferenciación social o por algún otro factor» ${ }^{34}$.

En este proceso es fundamental la figura retórica de la ironía que por su capacidad intertextual pone en relación los hipotextos latinos con el conjunto lingüístico contemporáneo en un continuo juego lingüístico con la finalidad de llamar la atención de un público ya aburrido por el allanamiento de la lengua cuotidiana.

\section{Referencias bibliográficas}

AA.VV. (2005): «Nunc est bibendum!!.». Un mito gráfico desde 1898, Michelin España Portugal.

AbruZzeSE, A. y Colombo, F. (ed. 1994): Il dizionario della pubblicità. Storie tecniche personaggi, Bologna, Zanichelli.

BACHTIN, M. (1975): Vroposy literatury i estetiki, Movska, Chudozestvennaja literatura; trad. it. (1979): Estetica e romanzo, Torino, Einaudi.

BALLIO, L. y RANOCCHI, A. (2009): Carosello story. La via italiana alla pubblicità televisi$v a$, Roma, Rai Radiotelevisione italiana.

CHIERICI, S. (29/09/2005): «Latino e automobili di oggi», Virtualcar, http://www.virtual car.it/archivionews/?n=209. Web visitada el 28/1/2012.

COTARD, R. (1925): «Le latin dans le réclame», Revue universitaire, XXXIV a., 216-218.

DARMON, O. (1997): El gran siglo de Bibendum, Paris, Ediciones Hoëbeke.

DE MARTINO, D. (2011): «Io sono Giulietta». Letterature \& miti nella pubblicità di auto, Bari, Levante.

- (2012): «El logos femenino en la publicidad mitólogica», en De Martino, F. y Morenilla Talens, M. (eds.), Teatro y sociedad: El logos femenino en el teatro, Bari, Levante, 325348.

De MarTino, F. (2010): Antichità \& pubblicità, Bari, Levante.

DEvYNCK, T. (2005): «Bibendum y los suyos», en Aa.Vv. (2005), op. cit., 23-35.

Fo, A. (2008): «Modi oraziani di pensare il tempo: tratti della fortuna moderna del carpe diem e di altri spunti delle Odi», en Audano, S. (ed.), Aspetti della Fortuna dell'Antico nella Cultura Europea. Atti della quinta giornata di studi, Sestri Levante, 7 marzo 2008 per Emanuele Narducci. Pisa, Edizioni ETS, 61-107.

FORIERO, E. (1999): Gli automobili. Il lessico delle prime quattro-ruote tra Ottocento e Novecento, Venezia, Marsilio.

GeNetTe, G. (1982): Palinsesti. La letteratura al secondo grado, Torino, Einaudi, 1997.

Grassi, G. (2010): «Un'acqua ad hoc», Alipes. Arte e cultura nella pubblicità, http://www.italipes.com/artelatino.htm. Web visitada el 28/1/2012.

${ }^{34}$ Bachtin, M. (1975): Vroposy literatury i estetiki, Movska, Chudozestvennaja literatura; trad. it. (1979): Estetica e romanzo, Torino, Einaudi, 166. Cfr. Ponzio, A. (2005): Testo come ipertesto e traduzione letteraria, Rimini, Guaraldi; PonZIO, A. (2009): Rencontres de paroles, Parigi, Alain Baudry \& Cie y Ponzio, A. (2010): Il linguaggio e le lingue, nueva ed., Bari, Graphis. 
PÉREZ, C. (2005): «Bibendum y las vanguardias (algunas consideraciones)», en Aa.Vv. (2005), op. cit., 7-11.

PONZIO, A. (2005): Testo come ipertesto e traduzione letteraria, Rimini, Guaraldi.

- (2009): Rencontres de paroles, Parigi, Alain Baudry \& Cie.

- (2010): Il linguaggio e le lingue, nuova ed., Bari, Graphis.

TosI, R. (1991): Dizionario delle sentenze latine e greche, Milano, Rizzoli.

SPINA, L. (1993): «La sententia nell'epoca della sua riproducibilità tecnica», en Pennacini, A. (ed.), Retorica e comunicazione: teoria e pratica della persuasione nella società contemporanea, Alessandria, Dell'Orso, 153-66.

Recibido: 5 de marzo de 2012

Aceptado: 29 de octubre de 2012 